\title{
Order Reduction in Optimal Event-triggered Control Design for Linear Stochastic Systems
}

\author{
Adam Molin, Henning Tischer and Sandra Hirche
}

\begin{abstract}
This paper considers the optimal design of eventtriggered controllers under a non-traditional average-cost criterion with costly observations. Determining the optimal eventtriggering law can be cast in the dynamic programming framework. Due to the lack of a closed form solution for the value function associated with the dynamic program, the methods for calculating the optimal solution suffer from the curse of dimensionality. Based on structural properties of the optimal solution, we develop a novel approximative method to reduce the dimensionality of the underlying optimization problem from the state dimension of the regulated process to the number of control inputs. As processes often consist of only few inputs compared to the number of state variables, such approach reduces the computational complexity significantly. It is shown that the proposed approximative event-trigger preserves the asymptotic behavior of the closed-loop system. A conditions is derived, when the reduced event-triggering law equals the optimal solution. We propose a measure to evaluate the approximation accuracy of the developed order reduction method.

Numerical simulations illustrate the obtained results and validate the effectiveness of the proposed model reduction method compared to the optimal solution.
\end{abstract}

\section{INTRODUCTION}

Recent advances in sensing, communications and embedded systems have shifted the paradigms in the design of distributed complex control systems. Therein, the efficient utilization of communication and computational capabilities is one of the key factors to increase control performance. Examples for such systems can be found in a variety of applications, including environmental monitoring, transportation networks and health care systems. In each of these systems, the sensing and controlling components are spatially distributed and exchange information over a resource constrained network. It has been shown by various researchers that event-triggered exchange of information is more suitable than time-triggered schemes, when resource constraints have to be considered [1]-[4].

The design approach for event-triggered control and estimation are often posed as optimization problems [5]-[11]. These are usually formulated in the framework of Markov decision processes and solved by dynamic programming. Although the underlying stochastic system is often assumed to be linear and the cost function has a quadratic form, the value function associated with the dynamic program does not admit a closed-form solution in general due to the non-standard

A. Molin, H. Tischer and S. Hirche are with the Institute of Automatic Control Engineering, Technische Universität München, Arcisstraße 21, D-80290 München, Germany; http://www. lsr.ei.tum.de, adam.molinetum.de, hirche@tum.de communication constraints. Brownian processes with initial state zero and constrained number of transmissions are an exception and have an analytical solution [5]. Hence, one is in general restrained to discretize the state space and apply the standard policy or value iteration [12]. As the number of discretized grid points grows exponentially with the dimensionality of the state space, such methods are only applicable in practice if the number of state variables is small. One way to counteract the curse of dimensionality is to approximate the value function by a parameterized function. In the context of event-triggered control, such approach has been proposed in [10] using quadratic approximate value functions. In this paper, we follow a different approach. The idea is to reduce the state space to the subspace that is most relevant for the event-trigger.

This paper addresses the problem of event-triggered control design for linear stochastic systems. The goal is to find an event-triggered controller that meets the trade-off between control performance and the average number of transmissions from sensor to controller. Such trade-off is reflected in an average-cost criterion, where the per-stage cost consists of a quadratic control cost and a communication penalty. The cost-function is inspired by work in [8] that derives optimal event-triggering schemes for an estimator-based networked control system. The main contribution of this paper is twofold. Built on results in [7] for finite horizon, we first show that, under mild conditions, the calculation of the optimal event-triggered controller for the average-cost criterion can be separated into standard subproblems. Second, we show that the structure of the optimal event-trigger admits an approximative model-order reduction, where the reducedorder system has a dimension equal to the number of control inputs. As processes often consist of only few inputs compared to the number state variables, such approach reduces the computational complexity significantly. In some cases, the reduced event-triggering law equals the optimal solution. A condition for this is derived and we propose a measure to evaluate the approximation accuracy of the developed order reduction method. Furthermore, we present numerical examples to evaluate the performance of our method.

The remainder of this paper is organized into four sections. In section II, we introduce the stochastic system model and describe the problem setting. Section III contains the main results of this paper and is divided into two subsections. First, we derive structural properties of the optimal solution and analyze the asymptotic behavior of the closed-loop system. Second, the order-reduction method is developed. In section IV, numerical simulations are conducted to validate 
the proposed method.

Notation. In this paper, the operators $\operatorname{tr}[\cdot]$ and $(\cdot)^{\mathrm{T}}$ denote the trace and the transpose operator, respectively. The expectation operator is denoted by $\mathrm{E}[\cdot]$ and the conditional expectation is denoted by $\mathrm{E}[\cdot \mid \cdot]$. The null-space of a matrix $A$ is denoted by null $[A]$ and the range of a Matrix $A$ is denoted by $\operatorname{span}[A]$. The Euclidean norm of a vector and the matrix norm induced by the Euclidean norm are denoted by $\|\cdot\|_{2}$.

\section{SYSTEM MODEL}

We consider the following stochastic time-invariant discrete-time system $\mathcal{P}$

$$
x_{k+1}=A x_{k}+B u_{k}+w_{k},
$$

where $A \in \mathbb{R}^{n \times n}, B \in \mathbb{R}^{n \times d}$. The variables, $x_{k}$ and $u_{k}$ denote the state and the control input and are taking values in $\mathbb{R}^{n}$ and $\mathbb{R}^{d}$, respectively, the system noise $w_{k}$ takes values in $\mathbb{R}^{n}$ and is an i.i.d. (independent identically distributed) zero-mean Gaussian distributed sequence with positive definite covariance matrix $C_{w}=\mathrm{E}\left[w_{k} w_{k}^{\mathrm{T}}\right]$. The initial state, $x_{0}$ is Gaussian with mean $\bar{x}_{0}$ and covariance $C_{x_{0}}=\mathrm{E}\left[\left(x_{0}-\bar{x}_{0}\right)\left(x_{0}-\bar{x}_{0}\right)^{\mathrm{T}}\right]$.

System parameters and statistics are known to the eventtrigger and controller. It is assumed that the event-trigger $\mathcal{E}$, situated at the sensor side, can observe the complete state and decides, whether the controller $\mathcal{C}$ should be updated with the current state. The controller is assumed to be situated at the actuator side, which implies that only sensor and controller must exchange information over the network. The system model is illustrated in Figure 1. The event-trigger output given by

$$
\delta_{k}= \begin{cases}1 & \text { update } x_{k} \text { sent } \\ 0 & \text { otherwise }\end{cases}
$$

Hence, the interconnection of sensor and controller can be stated as the following measurement equation, which differs from the standard LQG formulation:

$$
y_{k}= \begin{cases}x_{k} & \delta_{k}=1 \\ \emptyset & \delta_{k}=0\end{cases}
$$

where $y_{k}$ is the actual observation of the controller at time $k$.

The design objective is to find admissible control and event-triggering policies that minimize the average-cost criterion

$$
J=\limsup _{N \rightarrow \infty} \frac{1}{N} \mathrm{E}\left[\sum_{k=0}^{N-1} x_{k}^{\mathrm{T}} Q x_{k}+u_{k}^{\mathrm{T}} R u_{k}+\lambda \delta_{k}\right],
$$

whose per-stage cost is composed of a quadratic control cost term $x_{k}^{\mathrm{T}} Q x_{k}+u_{k}^{\mathrm{T}} R u_{k}$ and a communication cost term $\lambda \delta_{k}$. The weighting matrix $Q$ is positive definite and $R$ is positive semi-definite. The positive factor $\lambda$ can be regarded as the weight of penalizing information exchange between sensor and controller. We assume that the pair $(A, B)$ is controllable and the pair $\left(A, Q^{\frac{1}{2}}\right)$ is observable with $Q=\left(Q^{\frac{1}{2}}\right)^{\mathrm{T}} Q^{\frac{1}{2}}$. In addition, it is assumed that the control policy and eventtriggering policy are stationary.

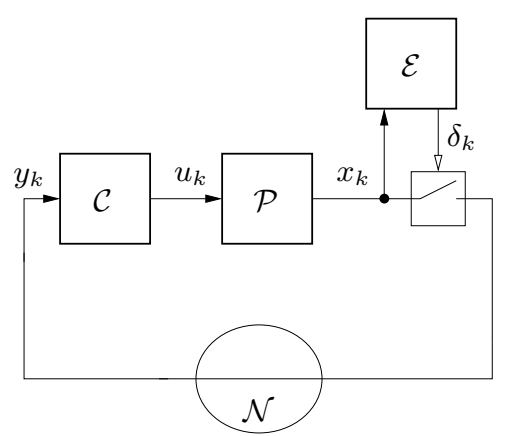

Fig. 1. System model of the networked control system with plant $\mathcal{P}$, event-trigger $\mathcal{E}$, controller $\mathcal{C}$ and communication network $\mathcal{N}$.

\section{MAIN RESULTS}

\section{A. Asymptotic behavior}

Before studying model reduction of the proposed problem, this section describes the structure of the optimal solution that minimizes the cost $J$ given by (3) and analyzes its asymptotic behavior. Finding the optimal policies that minimize the cost function in (3) is a very hard problem. This is due to the fact that the controller and event-trigger can be of a very general form just restricting them to be causal and stationary. In addition, the different information patterns [13] of event-trigger and controller prohibit a direct use of dynamic programming. However, it is shown in [7] that minimizing the cost function given by (3) for a finite horizon $N$ can be divided into separate subproblems. Therein, the optimal control law is related to linear quadratic regulation and the event-triggering law can be posed in the framework of dynamic programming.

With the mild restriction to stationary policies, the reformulation techniques developed in [7] for finite horizon problems also apply for the average-cost problem.

We then have the following theorem that facilitates our subsequent analysis.

Theorem 1 ( Structure of the optimal controller [7]):

Let the event-trigger and controller be causal and stationary. Then, the optimal control law minimizing (3) is given by

$$
u_{k}=-L \hat{x}_{k}^{\mathcal{C}}
$$

where

$$
\begin{aligned}
& L=-\left(B^{\mathrm{T}} S B+R\right)^{-1} B^{\mathrm{T}} S A \\
& S=A^{\mathrm{T}}\left(S-S B\left(B^{\mathrm{T}} S B+R\right)^{-1} B^{\mathrm{T}} S\right) A+Q .
\end{aligned}
$$

The least-squares estimate $\hat{x}_{k}^{\mathcal{C}}$ at the controller is given by

$$
\hat{x}_{k}^{\mathcal{C}}= \begin{cases}x_{k} & \delta_{k}=1 \\ (A-B L) \hat{x}_{k-1}^{\mathcal{C}} & \delta_{k}=0\end{cases}
$$

with $\hat{x}_{0}^{\mathcal{C}}=\bar{x}_{0}$ for $\delta_{0}=0$.

Using the optimal control law stated in Theorem 1 and an identity presented in [14], the per-stage cost in (3) reduces to

$$
\left(x_{k}-\hat{x}_{k}^{\mathcal{C}}\right)^{\mathrm{T}} L^{\mathrm{T}}\left(R+B^{\mathrm{T}} S B\right) L\left(x_{k}-\hat{x}_{k}^{\mathcal{C}}\right)+\lambda \delta_{k}
$$


We define the matrix

$$
\Gamma=L^{\mathrm{T}}\left(R+B^{\mathrm{T}} S B\right) L
$$

and the estimation error $e_{k}$ at the controller conditioned on $\delta_{k}=0$

$$
e_{k}=x_{k}-\mathrm{E}\left[x_{k} \mid \hat{x}_{k-1}^{\mathcal{C}}, \delta_{k}=0\right] .
$$

By definition of $\Gamma$ and $e_{k}$, the optimization problem can be formulated within the dynamic programming framework as follows. Consider the discrete-time system

$$
e_{k+1}=g\left(e_{k}, \delta_{k}, w_{k}\right)=\left(1-\delta_{k}\right) A e_{k}+w_{k}
$$

with initial condition $e_{0}=x_{0}-\bar{x}_{0}$. The remaining objective is to find the optimal event-triggering law $\pi^{*}$ defined by

$$
\delta_{k}=\pi^{*}\left(e_{k}\right)
$$

among all measurable mappings of the estimation error $e_{k}$ on $\{0,1\}$ that minimizes

$$
J^{\mathcal{E}}=\limsup _{N \rightarrow \infty} \frac{1}{N} \mathrm{E}\left[\sum_{k=0}^{N-1}\left(1-\delta_{k}\right) e_{k}^{\mathrm{T}} \Gamma e_{k}+\lambda \delta_{k}\right] .
$$

Remark 1: It can be observed immediately that $J^{\mathcal{E}}$ is nonnegative, as $\Gamma$ is positive semi-definite, and the minimum of $J^{\mathcal{E}}$ is upper-bounded by $\lambda$, as transmission on every time step is an admissible law yielding average-cost $J^{\mathcal{E}}=\lambda$.

The above average-cost problem differs from the problem statement in [8] by the fact that $\Gamma$ is not positive definite if the number of inputs is less than the dimensionality of the state space. In the following, we are interested in this particular case of $d<n$. In this case, there exists a subspace of $\mathbb{R}^{n}$, where $e_{k}$ does not contribute to the per-stage cost. This subspace is spanned by the null-space of $\Gamma$. As it is explained in the next subsection in more detail, the optimal policy $\pi^{*}\left(e_{k}\right)$ does not transmit information, if $e_{k}$ is in the null-space of $\Gamma$. In contrast to that, the work in [8] restricts admissible event-triggering policies to transmit an update, whenever the norm of $e_{k}$ exceeds an arbitrary large positive constant. The restriction is a technical assumption to guarantee that the per-stage cost is bounded, which facilitates the analysis. In order to obtain similar results for the following analysis, we modify the set of admissible policies to satisfy

$$
\pi\left(e_{k}\right)=1 \text { for }\left\|L e_{k}\right\|_{2} \geq M,
$$

where $M$ is a positive constant. The condition (7) ensures that the per-stage cost of $J^{\mathcal{E}}$ defined in (6) is bounded, while allowing policies with $\pi\left(e_{k}\right)=1$ for $e_{k} \in \operatorname{null}[\Gamma]$. As $M$ can be chosen arbitrarily large, the condition (7) does not constitute a severe restriction on the optimal policy. Along the same lines as in [8], we can state that the optimal policy $\pi^{*}$ results from the Average-Cost Optimality Equation [15]

$$
\begin{aligned}
h(e)+J^{\mathcal{E}^{*}}=\min _{\delta \in\{0,1\}}( & (1-\delta) e^{\mathrm{T}} \Gamma e+\lambda \delta \\
& +\mathrm{E}[h(g(e, \delta, w)) \mid e, \delta]),
\end{aligned}
$$

where $e \in \mathbb{R}^{n}$ and the bounded function $h: \mathbb{R}^{n} \rightarrow \mathbb{R}$ is called the terminal cost. The value $J^{\mathcal{E}^{*}}$ is the optimal average-cost. The optimal policy can be computed by application of value or policy iteration [12].

We finish this paragraph by analyzing the stability properties of the process with state $x_{k}$ when applying the optimal controller and event-trigger. Subsequently, the notion of stability is given in terms of bounded moment. For that reason, we give the difference equation of the closed-loop system in the following rewritten form

$$
x_{k+1}=(A-B L) x_{k}+\left(1-\pi^{*}\left(e_{k}\right)\right) B L e_{k}+w_{k}
$$

with appropriate initial condition. The stochastic process $\left(1-\pi^{*}\left(e_{k}\right)\right) B L e_{k}$ has a bounded support for every $k$ because of (7). In fact, there exists a uniform bound for the support due to Equation (7). Therefore, the augmented system noise $\left(1-\pi^{*}\left(e_{k}\right)\right) B L e_{k}+w_{k}$ has a bounded second moment uniformly in $k$. As the matrix $(A-B L)$ is Hurwitz [14], it can be concluded that the process state $x_{k}$ has a bounded second moment for $k \rightarrow \infty$. The state estimate $\hat{x}_{k}^{\mathcal{C}}$ at the controller can be regarded as an asymptotically stable system, where the initial condition is reset to $x_{k}$, if $\delta_{k}=1$. Therefore, bounded moment stability of $x_{k}$ also implies that $\hat{x}_{k}^{\mathcal{C}}$ is stable in terms of bounded moment. Since the evolution of the overall system can be described by the two variables $x_{k}$ and $\hat{x}_{k}^{\mathcal{C}}$, we conclude that the closed-loop system is bounded moment stable.

\section{B. Order Reduction}

The calculation of the optimal control gain $L$ is of minor computational complexity compared to the problem of finding the optimal event-triggering law $\pi^{*}$. Therefore, our focus is on the numerically efficient calculation of $\pi^{*}$. This section develops an approach for diminishing computational complexity to find $\pi^{*}$ by model order reduction. We will observe in the following that the underlying problem admits an order reduction to the number of inputs, while approximating the optimal solution very closely. In some situations, it is even possible to sustain the optimal solution through order reduction.

The main idea is based on the observation that the weighting matrix $\Gamma$ is of reduced rank, if $d<n$. Hence, only a projected part of the estimation error $e_{k}$ contributes to the per-stage cost given by (6). On the other hand, the projected signal of $e_{k}$ in the null-space of $\Gamma$ may still influence the evolution of the complete signal $e_{k}$. Our conjecture is that the impact of $e_{k}$ in the null-space of $\Gamma$ is negligible for most systems.

In order to obtain the reduced optimization problem, we consider the eigenvalue decomposition of $\Gamma$

$$
\Gamma=\left[\begin{array}{cc}
U_{\|} & U_{\perp}
\end{array}\right]\left[\begin{array}{cc}
\Delta & 0 \\
0 & 0
\end{array}\right]\left[\begin{array}{c}
U_{\|}^{\mathrm{T}} \\
U_{\perp}^{\mathrm{T}}
\end{array}\right],
$$

where $\Delta$ contains all non-zero eigenvalues with corresponding eigenspace $U_{\|}$; the null-space of $\Gamma$ corresponds to $\operatorname{span}\left[U_{\perp}\right]$. The matrix $\left[U_{\|} U_{\perp}\right]$ is chosen to be orthonormal. 
We split up the estimation error into two components $e_{k}^{\perp}$ and $e_{k}^{\|}$, i.e.

$$
e_{k}=e_{k}^{\perp}+e_{k}^{\|},
$$

where $e_{k}^{\perp} \in \operatorname{null}[\Gamma]$ and $e_{k}^{\|} \in \operatorname{span}\left[\Gamma^{\mathrm{T}}\right]$. Only the signal $e_{k}^{\|}$ contributes to the per-stage cost of $J^{\mathcal{E}}$ given by (6). Subsequently, we propose an approximative model of the evolution of $e_{k}^{\|}$in a lower dimensional space using orthogonal projection. The reduced estimation error is defined as

$$
e_{k}^{\mathrm{red}}=U_{\|}^{\mathrm{T}} e_{k}=U_{\|}^{\mathrm{T}} e_{k}^{\|}
$$

evolving according to the following dynamics

$$
e_{k+1}^{\mathrm{red}}=\left(1-\delta_{k}\right) A_{\mathrm{red}} e_{k}^{\mathrm{red}}+w_{k}^{\mathrm{red}},
$$

with

$$
\begin{gathered}
A_{\text {red }}=U_{\|}^{\mathrm{T}} A U_{\|}, \\
\quad w_{k}^{\mathrm{red}}=U_{\|}^{\mathrm{T}} w_{k}
\end{gathered}
$$

and initial state $e_{0}^{\mathrm{red}}=U_{\|}^{\mathrm{T}}\left(x_{0}-\bar{x}_{0}\right)$. The noise process $w_{k}^{\mathrm{red}}$ is Gaussian with zero-mean and covariance matrix $U_{\|}^{\mathrm{T}} C_{w} U_{\|}$.

Given the dynamics in (8) and the rewritten average-cost criterion from (6)

$$
J^{\mathcal{E}}=\limsup _{N \rightarrow \infty} \frac{1}{N} \mathrm{E}\left[\sum_{k=0}^{N-1}\left(1-\delta_{k}\right)\left(e_{k}^{\mathrm{red}}\right)^{\mathrm{T}} \Delta e_{k}^{\mathrm{red}}+\lambda \delta_{k}\right],
$$

the optimal solution $\pi_{\text {red }}^{*}\left(e_{k}^{\text {red }}\right)$ minimizing (9) yields an approximative solution to our initial problem. Taking the restriction given by Equation (7) into account, we observe that admissible event-triggering policies $\pi_{\text {red }}$ have to transmit an update to the controller, whenever the norm of $e_{k}^{\text {red }}$ exceeds the constant $M$. This implies that closed-loop stability is preserved, although the reduced difference equation in (8) may give a coarse approximation of the real estimation error $e_{k}$.

The structure of the reduced event-trigger is depicted in Figure 2. It consists of a copy of the state estimator at the controller, a one-step time-delay unit $\mathrm{T}$, transformation $U_{\|}^{\mathrm{T}}$ and optimal event-triggering law $\pi_{\text {red }}^{*}$. The difference between state estimate of the controller assuming no transmission has occurred at time $k$ and the current state is transformed by $U_{\|}^{\mathrm{T}}$ to obtain $e_{k}^{\text {red }}$. The reduced event-triggering law $\pi_{\text {red }}^{*}$ decides whether to update the controller with the current state $x_{k}$ based on the current reduced estimation error. It should be noted that the proposed scheme also reduces the online calculation of the decision variable $\delta_{k}$, as the dimensionality of the search space decreases to $d$. In contrast to that there is no benefit in memory usage, as both reduced and optimal event-triggers have to store the complete state estimate of the controller.

Remark 2: We observe from the definition of $\Gamma$ in (4) that its range coincides with the range of $L^{\mathrm{T}}$, as $\left(R+B^{\mathrm{T}} S B\right)$ is invertible. Therefore, the dimension of $\operatorname{span}[\Gamma]$ is equal to the column-rank of $L^{\mathrm{T}}$, which is at most $d$. This implies that the proposed method reduces the state space associated with the

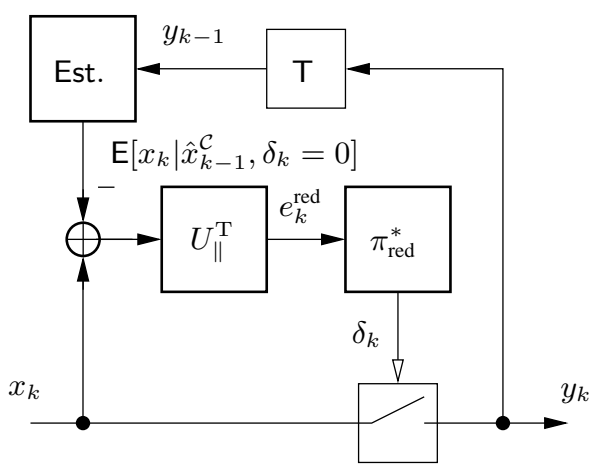

Fig. 2. Structure of the reduced event-trigger with a copy of the state estimator of the controller (Est.), a one-step time-delay unit T, transformation $U_{\|}^{\mathrm{T}}$ and optimal event-triggering law $\pi_{\text {red }}^{*}$.

dynamic program by at least $n-d$ dimensions. As technical processes often consist of only few inputs compared to the dimensionality of their state space, the underlying reduction algorithm is able to decrease computational complexity significantly for many technical systems.

Remark 3: It should be stated that the established theory of state projection methods in model order reduction, e.g. from [16], is of limited use in our context, because the system given by (5) is highly non-linear with respect to the decision variable $\delta_{k}$ and the system may be unstable without having stable modes. Another aspect, in which our approach differs from standard methods in model order reduction, is that asymptotic properties need not to be taken into account. This is reasoned by the resetting property of the underlying system, i.e. whenever $\delta_{k}=1$ the error $e_{k}$ is reset by $e_{k+1}=w_{k}$. Obviously, such resetting property is preserved by our order reduction.

The next lemma gives a condition, when the reduced policy recovers the optimal policy.

Lemma 1 (Perfect Reduction): If the projector $P$ defined by

$$
P=U_{\|} U_{\|}^{\mathrm{T}}
$$

and the system matrix $A$ satisfy

$$
U_{\|}^{\mathrm{T}} P A=U_{\|}^{\mathrm{T}} A P,
$$

then the optimal policy $\pi^{*}$ and the reduced optimal policy $\pi_{\text {red }}^{*}$ embedded in $\mathbb{R}^{n}$ are equivalent.

Proof: The evolution of the projected estimation error $e_{k}^{\text {red }}$ under the original dynamics given by (5) can be computed as

$$
\begin{aligned}
e_{k+1}^{\mathrm{red}} & =U_{\|}^{\mathrm{T}} e_{k+1} \\
& =\left(1-\delta_{k}\right) U_{\|}^{\mathrm{T}} A e_{k}+U_{\|}^{\mathrm{T}} w_{k} \\
& =\left(1-\delta_{k}\right) U_{\|}^{\mathrm{T}} U_{\|} U_{\|}^{\mathrm{T}} A e_{k}+U_{\|}^{\mathrm{T}} w_{k}
\end{aligned}
$$

Taking the condition of above lemma into account, we have

$$
e_{k+1}^{\mathrm{red}}=\left(1-\delta_{k}\right) U_{\|}^{\mathrm{T}} A U_{\|} U_{\|}^{\mathrm{T}} e_{k}+U_{\|}^{\mathrm{T}} w_{k},
$$

By substituting $e_{k}^{\text {red }}=U_{\|}^{\mathrm{T}} e_{k}$, we obtain the difference equation (8). Hence, the random variable $e_{k}^{\text {red }}$ is a sufficient 
statistics for obtaining the initial problem minimizing (6) under dynamics (5). This concludes the proof.

Remark 4: There is an intuitive interpretation why $\Gamma$ has a rank deficiency, when $d<n$. The null-space of $\Gamma$ is equivalent to the null-space of $L$. If $e_{k} \in \operatorname{null}[\Gamma]=\operatorname{null}[L]$, then the current control input $u_{k}$ does not differ, when an update is sent or not. Hence, the transmission can be postponed in case of $e_{k} \in \operatorname{null}[\Gamma]$ in order to yield better state estimates at a future time step.

Remark 5: The approach of model order reduction for event-trigger design has also been suggested in [11] for linear discrete-time systems with limited controls for the special case that the system matrix takes the form $\alpha I$, input matrix $B$ has full rank and the weighting matrix of the quadratic cost function is of rank 1 .

As commutativity of arbitrary matrices is a quite restrictive assumption, the condition given in Lemma 1 holds only for specific systems. But it can serve as an indicator for how well the optimal event-triggering law is approximated by the reduced policy. In this work, we propose the following measure to give an indicator of the approximation accuracy of the order-reduced algorithm

$$
\rho=\frac{\left\|U_{\|}^{\mathrm{T}}(A P-P A)\right\|_{2}}{\|A\|_{2}}
$$

assuming the system matrix $A$ to be non-zero. When $d<n$, the assumption of $A \neq 0$ is already covered by the controllability assumption of the tuple $(A, B)$. Obviously, the measure $\rho$ is non-negative. The measure $\rho$ is equal to 0 , if and only if the condition in Lemma 1 is satisfied. This means that we have perfect reduction for $\rho=0$. On the other hand, the measure $\rho$ is bounded by 1 as shown in the following

$$
\begin{aligned}
\rho & =\frac{\left\|U_{\|}^{\mathrm{T}}(A P-P A)\right\|_{2}}{\|A\|_{2}} \\
& =\frac{\left.\| U_{\|}^{\mathrm{T}} A P-U_{\|}^{\mathrm{T}} U_{\|} U_{\|}^{\mathrm{T}} A\right) \|_{2}}{\|A\|_{2}} \\
& =\frac{\left\|U_{\|}^{\mathrm{T}}(A P-A)\right\|_{2}}{\|A\|_{2}} \\
& \leq \frac{\left\|U_{\|}^{\mathrm{T}}\right\|_{2}\|A\|_{2}\|P-I\|_{2}}{\|A\|_{2}}=1 .
\end{aligned}
$$

\section{NUMERICAL VALIDATION}

In this section, we validate our results from the previous section by numerical simulations. Two different systems with a 2-dimensional state space and a scalar input are considered. Figures 3 and 5 illustrate the effect of model order reduction on the resulting event-trigger. The coordinates $e_{1}$ and $e_{2}$ are the elements of the estimation error $e$. If $e$ exceeds the indicated thresholds, a state update is sent to the controller. The system underlying Figure 3 is given by

$$
\begin{aligned}
& A=\left[\begin{array}{cc}
0.65 & 0.95 \\
0.5 & 0.15
\end{array}\right], B=\left[\begin{array}{l}
0.7 \\
0.7
\end{array}\right], Q=Q_{N}=\left[\begin{array}{ll}
2 & 1 \\
1 & 2
\end{array}\right], \\
& R=0.5, C_{\omega}=C_{x_{0}}=\left[\begin{array}{ll}
1.5 & 0.5 \\
0.5 & 1.5
\end{array}\right], \lambda=200,
\end{aligned}
$$

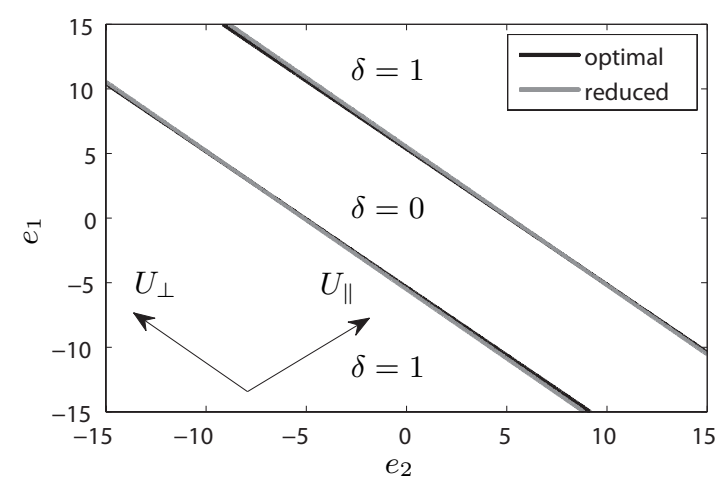

Fig. 3. Comparison of optimal and reduced event-triggering policy of system (11). The lines indicate the thresholds.

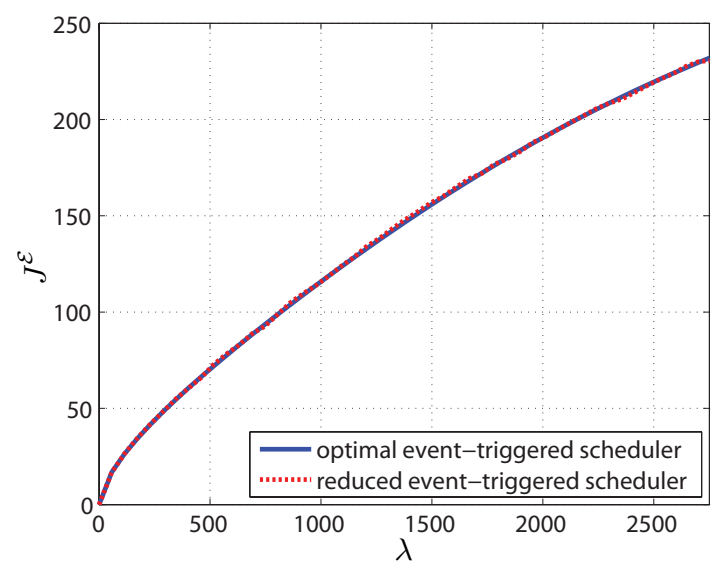

Fig. 4. Performance evaluation of optimal and reduced event-triggering policy of system (11).

and the reduced event-trigger obviously approximates the optimal event-trigger very well. Figure 4 shows the per-stage cost caused by the optimal event-trigger and the reduced event-trigger.

Figure 5 shows the same comparison for the system

$$
\begin{aligned}
& A=\left[\begin{array}{cc}
0 & -1 \\
1 & 0
\end{array}\right], B=\left[\begin{array}{l}
0.7 \\
0.7
\end{array}\right], Q=Q_{N}=\left[\begin{array}{ll}
2 & 1 \\
1 & 2
\end{array}\right], \\
& R=0.5, C_{\omega}=C_{x_{0}}=\left[\begin{array}{ll}
1.5 & 0.5 \\
0.5 & 1.5
\end{array}\right], \lambda=100 ;
\end{aligned}
$$

There is a significant difference in the optimal eventtrigger and the reduced event-trigger. Figure 6 shows the 


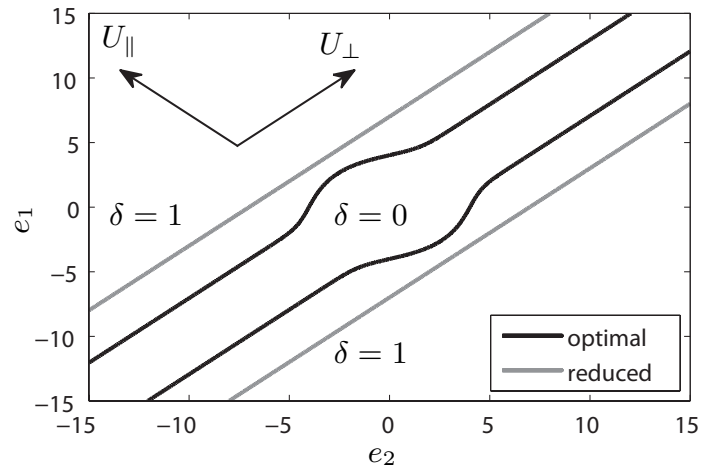

Fig. 5. Comparison of optimal and reduced event-triggering policy of system (12). The lines indicate the thresholds.

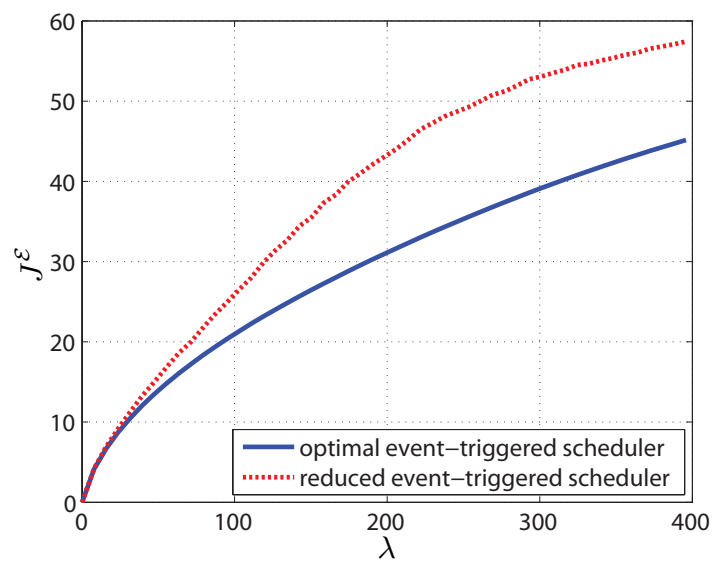

Fig. 6. Performance evaluation of optimal and reduced event-triggering policy of system (12).

per-stage costs caused by both event-triggers for various $\lambda$ and it is obvious that the proposed approach does not work very well for this system. These numerical results are also in accordance with the proposed indicator $\rho$ with respect to the approximation quality. While system (11) yields a $\rho$ of 0.12 , which is an indicator for an accurate approximation of the optimal solution, system (12) has a $\rho$ of 1 , which can be regarded as the worst case setting with respect to $\rho$.

The jittering of the graph for $J^{\mathcal{E}}$ caused by the reduced event-trigger in Figures 4 and 6 is due to the fact that $J^{\mathcal{E}}$ is determined with a Monte Carlo experiment for this eventtrigger.

It should be stated that a series of simulations with randomly generated systems has been conducted to validate the presented approach. Most of these systems show similar approximation accuracy as given for system (11), whereas the resulting approximation quality of system (12) is observed only sparsely.

\section{CONCLUSIONS}

This paper derives a novel method for the approximative design of optimal event-triggered controllers. In contrast to standard approaches to overcome the curse of dimensionality, this method takes advantage of the structure within the underlying problem. Our results show that this method seems to be very promising, if the number of process inputs is smaller than the dimension of states. Another benefit of the approximative approach is that stability is preserved. The proposed indicator for the approximation quality of the reduced event-trigger reflects the performance decrease very accurately.

Future research is concerned with advanced order reduction schemes that enhance the approximation accuracy of the reduced event-trigger and with the investigation of bounds on the performance decrease of the approximative scheme.

\section{ACKNOWLEDGMENTS}

This work was supported in part by the German Research Foundation (DFG) within the Priority Program SPP 1305 "Control Theory of Digitally Networked Dynamical Systems".

\section{REFERENCES}

[1] K. Åström and B. Bernhardsson, "Comparison of Riemann and Lebesgue sampling for first order stochastic systems," in Decision and Control, 2002, Proceedings of the 41st IEEE Conference on, 2002.

[2] A. Cervin and T. Henningsson, "Scheduling of event-triggered controllers on a shared network," in Decision and Control, 2008, Proceedings of the 47th IEEE Conference on, (Cancun, Mexico), 2008.

[3] P. Tabuada, "Event-triggered real-time scheduling of stabilizing control tasks," Automatic Control, IEEE Transactions on, vol. 52, no. 9, pp. $1680-1685,2007$.

[4] X. Wang and M. Lemmon, "Event design in event-triggered feedback control systems," in Decision and Control, 2008, Proceedings of the 47th IEEE Conference on, (Cancun, Mexico), 2008.

[5] M. Rabi, K. H. Johansson, and M. Johansson, "Optimal stopping for event-triggered sensing and actuation," in Decision and Control, 2008, Proceedings of the 47th IEEE Conference on, (Cancun, Mexico), 2008.

[6] O. C. Imer and T. Basar, "To measure or to control: optimal control with scheduled measurements and controls," American Control Conference, 2006. Proceedings of the 2006, pp. 1003-1008, June 2006.

[7] A. Molin and S. Hirche, "On LQG joint optimal scheduling and control under communication constraints," in Decision and Control, 2009, Proceedings of the 48th IEEE Conference on, (Shanghai, China), 2009.

[8] Y. Xu and J. Hespanha, "Optimal communication logics in networked control systems," in Decision and Control, 2004, Proceedings of the 43rd IEEE Conference on, (Nassau, Bahamas), 2004.

[9] Y. Xu and J. P. Hespanha, "Communication logic design and analysis for networked control systems," in Current trends in nonlinear systems and control (L. Menini, L. Zaccarian, and C. T. Abdallah, eds.), Boston: Birkhäuser, 2006.

[10] R. Cogill, "Event-based control using quadratic approximate value functions," in Decision and Control, 2009, Proceedings of the 48th IEEE Conference on, (Shanghai, China), 2009.

[11] P. Bommannavar and T. Başar, "Optimal control with limited control actions and lossy transmissions," in Decision and Control, 2008, Proceedings of the 47th IEEE Conference on, (Cancun, Mexico), 2008.

[12] D. P. Bertsekas, Dynamic programming and optimal control. Vol. II 3rd ed., Athena Scientific, Belmont, MA, 2007.

[13] H. Witsenhausen, "On information structures, feedback and causality," SIAM Journal on Control, vol. 9, p. 149, 1971.

[14] K. J. Åström, Introduction to Stochastic Control Theory. Dover Publications, 2006.

[15] O. Hernández-Lerma and J. Lasserre, "Discrete-time Markov Control Processes," Appl. Math, vol. 30, 1996.

[16] G. Obinata and B. Anderson, Model reduction for control system design. Springer Verlag, 2001. 